\title{
RELATIONSHIP BETWEEN FLORAL MORPHOLOGY, FRUIT SETTING BEHAVIOR AND FINAL YIELD IN SOME EGGPLANT (Solanum melongena) GENOTYPES FROM IRAN
}

\author{
Sedighehsadat Khaleghi ${ }^{1 \mathrm{a} *}$, Bahram Baninasab ${ }^{1 \mathrm{~b}}$, Mostafa Mobli $^{1 \mathrm{c}}$ \\ ${ }^{1 a}$ Department of Horticulture, College of Agriculture, Isfahan University of Technology, Isfahan, Iran. \\ https://orcid.org/0000/0001/5425/6456 \\ ${ }^{1 b}$ Department of Horticulture, College of Agriculture, Isfahan University of Technology, Isfahan, Iran. \\ https://orcid.org/0000/0002/9138/2221 \\ ${ }^{1 c}$ Department of Horticulture, College of Agriculture, Isfahan University of Technology, Isfahan, Iran. \\ https://orcid.org/0000/0003/2575/8427 \\ * Corresponding author E-mail: khaleghi1360@yahoo.com
}

\section{ABSTRACT}

Heterostyly in eggplant flowers is a common trait that may affect the fruit production. The objective of this study was to evaluate the impact of heterostyly on fruit setting and yield of $\mathbf{1 3}$ eggplant genotypes from Iran. Flowers capable of setting fruit, including long- (LGs) and medium-styled flowers (MEs), accounted for the largest number of flowers $(43.60-75.62 \%)$, while total short-styled flowers (SRTs) constituted a smaller percentage (20.47-45.51\%) in different genotypes. However, SRTs represented a considerable proportion of the total number of eggplant flowers. Different eggplant genotypes produced $42.59-77.25 \%$ fruits from LGs+MEs, and only $0.0-3.77 \%$ from SRTs. In general, although the percentage of fruit setting of LGs and MEs was much greater compared to values of SRTs, there was no significant correlation between final yield and style length. Therefore, in order to increase eggplant yield, protecting fruits formed from LGs and MEs might be more effective than increasing the proportion of LGs and MEs to SRTs.

Key words: abscission, eggplant, final fruit set, heterostyly, initial fruit set

\section{INTRODUCTION}

Eggplant (Solanum melonegena L.) is one of the most widely grown species of the Solanaceae family in both tropical and subtropical regions (San José et al., 2016). Its total world annual production reached over 55 million tons in 2019, with an annual production of 670158 tons. Iran is the fifth leading eggplant producer, after China, India, Egypt and Turkey (FAOSTAT, 2019). Eggplant fruits are rich in phenolic compounds and flavonoids, showing a high antioxidant and oxygen radical absorbance capacity (Singh et al., 2006; Raigón et al., 2008; Caruso et al., 2017).

Eggplant flowers are large and usually violetcolored. They consist of five united and persistent sepals, five united and cup-shaped petals, five stamens alternating with the corolla, united carpels, and superior ovaries arranged either singly or in inflorescence (Rashid and Singh, 2000; Hazra et al., 2003; Jagatheeswari, 2014). The number of flower buds varies in inflorescences, ranging between 2 and 7 (Hazra et al., 2003). A specific form of heterostyly has been reported for eggplant, in which flowers have different style lengths in the same plant (Prasad and Prakash, 1968; Rylski et al.,1984; Sękara and Bieniasz, 2008). In this sense, eggplant flowers have been classified into four groups based on the style to stamen length ratio: long-styled flowers (LGs) with large ovaries, medium-styled (MEs) with medium-sized ovaries, pseudo short-styled 
flowers (PSs) with rudimentary ovaries, and true short-styled flowers (TSs) with very rudimentary ovaries (Krishnamurtbi and Subramanian, 1954). Several researchers have studied the relationship between fruit set and stigma position. High fruit set rates have been reported in LGs and MEs, but severe fertility and fruit set problems have been found in SRTs (PS+TS), with flowers that are not capable of setting fruits or have low quality and small fruits (Srinivas et al., 2016; Pohl et al., 2019).

Studies on heterostyly in eggplant were conducted until 2000, reporting percentages of flowers based on style length and the relationship between fruit setting behavior and types of flowers in some eggplant cultivars (Rylski et al., 1984; Sękara and Bieniasz, 2008; Srinivas et al., 2016; Pohl et al., 2019). However, to the best of our knowledge, the direct relationship between these types of flowers and final yield has not yet been reported. Therefore, the objective of this study was to determine the percentages of flowers capable (LGs+MEs) and incapable of setting fruits (SRTs), as well as fruit set rates of these kinds of flowers in 13 eggplant genotypes from Iran, with emphasis on fruit set of single flowers or inflorescences. Moreover, final fruit set and final yield were also evaluated in order to determine the correlation between final yield and flower style length.

\section{MATERIALS AND METHODS}

\section{Plant materials}

Thirteen S. melongena genotypes, obtained from the National Plant Gene Bank at the Seed and Plant Improvement Institute, Karaj, Iran, were used (Table 1). Seeds of each genotype were sown in the middle of March 2017 into the 1 -L pots $(13 \times 11 \mathrm{~cm})$ filled with peat and perlite substrate at a ratio of $4: 1 ; \mathrm{v} / \mathrm{v}$ and placed on a greenhouse bench. Greenhouse temperature was set at $20 / 28^{\circ} \mathrm{C}$ (night/day), and humidity of 60 $85 \%$. Six weeks after sowing, uniform seedlings were transplanted into the experimental field at the Isfahan University of Technology, Isfahan, Iran. This place is located at an altitude of 1624 $\mathrm{m}$, latitude $32^{\circ} 42^{\prime} \mathrm{N}$, longitude $51^{\circ} 28^{\prime} \mathrm{E}$, with an average of $159 \mathrm{~mm}$ annual rainfall and $18.2^{\circ} \mathrm{C}$ air temperature. The type of soil is fine-loamy, carbonatic and thermic typic haplocalcids. Spacing between the plants was $60 \times 60 \mathrm{~cm}$. The soil of the experimental site was sandy loam with organic matter content of $1.12 \%$ and nitrogen concentration of $0.03 \%$. The amount of clay, silt and sand were 33,17 and $50 \%$, respectively. Bulk density, field capacity, and $\mathrm{pH}$ of the soil reached $1.27 \mathrm{~g} / \mathrm{cm} 3,25 \%$ and 7.99 , respectively. Prior to cultivation, the field was ploughed with 25 tones ha $^{-1}$ of organic manure. Organic matter content, nitrogen concentration and $\mathrm{pH}$ of the ploughed soil were $1.78 \%, 0.07 \%$, and 7.50 , respectively. The Floral fertilizer 20-20-20, including NPK 20-20-20+B+Cu+Fe+Mn+Mo+Zn was also applied once a month at a concentration of $1 \mathrm{mg} \mathrm{L}^{-1}$, and one litre of this solution was given to each plant. The plants were drip-irrigated with an average flow rate of $2.4 \mathrm{~L} \mathrm{~h}^{-1}$. Pests and associated diseases were treated using chemical products during the growing season. Confidor (imidacloprid) and Oberon (spiromesifen) were applied against aphids and whitefly, respectively. Abamectin was applied against mites and thrips. Iprodionecarbendazim and copper oxychloride were used to control fungi.

Table 1. Genotypes of Solanum melongena and their origin.

\begin{tabular}{ccllcrr}
\hline Code & $\begin{array}{c}\text { Genotype } \\
\text { number }\end{array}$ & Fruit character & $\begin{array}{c}\text { Origin of the } \\
\text { material (Province) }\end{array}$ & Longitude & Latitude & Altitude (m) \\
\hline 1 & TN74116 & Long and slender & Khorasan & $54^{\circ} 18^{\prime} \mathrm{E}$ & $32^{\circ} 33^{\prime} \mathrm{N}$ & 1100 \\
2 & TN74238 & Egg-shaped & Hormozgan & $56^{\circ} 27^{\prime} \mathrm{E}$ & $27^{\circ} 18^{\prime} \mathrm{N}$ & 30 \\
3 & TN74197 & Egg-shaped & Isfahan & $51^{\circ} 67^{\prime} \mathrm{E}$ & $32^{\circ} 65^{\prime} \mathrm{N}$ & 900 \\
4 & TN74100 & Dwarf type & Ghazvin & $50^{\circ} 01^{\prime} \mathrm{E}$ & $36^{\circ} 27^{\prime} \mathrm{N}$ & 1280 \\
5 & TN74128 & Dwarf type & Kordestan & $46^{\circ} 90^{\prime} \mathrm{E}$ & $35^{\circ} 61^{\prime} \mathrm{N}$ & 555 \\
6 & TN74237 & Dwarf type & Hormozgan & $56^{\circ} 27^{\prime} \mathrm{E}$ & $27^{\circ} 18^{\prime} \mathrm{N}$ & 21 \\
7 & TN74156 & Egg-shaped & Kerman & $57^{\circ} 07^{\prime} \mathrm{E}$ & $30^{\circ} 28^{\prime} \mathrm{N}$ & 1756 \\
8 & TN74243 & Long and slender & Kordestan & $46^{\circ} 90^{\prime} \mathrm{E}$ & $35^{\circ} 61^{\prime} \mathrm{N}$ & 1000 \\
9 & TN74239 & Long and slender & Hormozgan & $56^{\circ} 27^{\prime} \mathrm{E}$ & $27^{\circ} 18^{\prime} \mathrm{N}$ & 30 \\
10 & TN74120 & Long and slender & East Azarbayejan & $56^{\circ} 27^{\prime} \mathrm{E}$ & $27^{\circ} 18^{\prime} \mathrm{N}$ & 1350 \\
11 & TN74250 & Long and slender & Zanjan & $48^{\circ} 30^{\prime} \mathrm{E}$ & $36^{\circ} 41^{\prime} \mathrm{N}$ & 1700 \\
12 & TN74161 & Long and slender & West Azarbayejan & $54^{\circ} 18^{\prime} \mathrm{E}$ & $32^{\circ} 33^{\prime} \mathrm{N}$ & 325 \\
13 & TN74231 & Long and slender & Kohgiluyeh & $50^{\circ} 40^{\prime} \mathrm{E}$ & $30^{\circ} 49^{\prime} \mathrm{N}$ & 600 \\
\hline
\end{tabular}




\section{Observations}

Observations of flower morphology and fruit setting were made on a daily basis from the beginning of June (70 days after sowing), and continued throughout the reproductive stage until the end of August (160 days after sowing). The following data about flowering and fruit setting were recorded for all genotypes: number of flowers in inflorescence; number of long-styled flowers (LGs), medium-styled flowers (MEs), pseudo short-styled flowers (PSs), and true short-styled flowers (TSs) in single flowers and inflorescences, separately; and number of LGs, MEs, PSs and TSs setting to fruit (initial number of fruit per plant). The length and thickness of pedicel were measured directly on the plant in the field in all types of flowers. Fruits were harvested at maturity stage, beginning in the first ten days of July. The number of fruits per plant (final fruit set) and final yield were estimated until the end of October.

\section{Data analysis}

The experiment was conducted in a randomized complete block design (RCBD) with a factorial arrangement (including types of flowers based on style length (LG, ME, PS and TS) as the main factor, and types of flowers based on their position on the plant (single flowers and inflorescences) as the secondary factor), with 3 replicates; each replicate contained 3 plants for each genotype. In addition, the number of flowers per inflorescence, pedicel length and thickness, final fruit set and yield were carried out based on a randomized complete block design. The results were analysed using an analysis of variance (ANOVA) using SAS software, and differences between means were compared using the least significant differences (LSD) test $(\mathrm{p}<0.05)$.

\section{RESULTS}

\section{Number of floral buds per inflorescence}

The number of flowers per inflorescence ranged from 2 to 6 , varying depending on each genotype (Table 2). Only genotypes TN74239 and TN74231 had a small percentage of 6-flowered inflorescence. Except for genotypes TN74197, TN74100 and TN74120, the rest of the genotypes had 5-flowered inflorescences, but with a very low percentage. Only genotype TN74120 did not have 4-flowered inflorescence. All the genotypes had high percentages of 2 and 3-flowered inflorescences, which were dominant in all genotypes, except for genotype TN74239 with the highest percentage of 4-flowered inflorescence.

\section{Types of flowers based on style length}

All the genotypes produced flowers of the four types (long, medium, pseudo and true shortstyled). Flower abscission occurred at a low rate prior to anthesis and style emergence (data not shown). In most genotypes, including TN74238, TN74197, TN74100, TN74156, TN74120, TN74250, TN74161 and TN74231, LGs of the single type account for most of the total number of flowers in a plant $(46.80,28.53,25.67,34.07,56.24,34.84,34.56$, and $26.67 \%$, respectively), followed by LGs and PSs in inflorescence. In the rest of the genotypes, including TN74116, TN74128, TN74237, TN74243

Table 2. Percentage of inflorescences in 13 eggplant genotypes.

\begin{tabular}{cccccc}
\hline Genotype & $\begin{array}{c}\text { 2- Flowered } \\
\text { inflorescence }\end{array}$ & $\begin{array}{c}\text { 3- Flowered } \\
\text { inflorescence }\end{array}$ & $\begin{array}{c}\text { 4- Flowered } \\
\text { inflorescence }\end{array}$ & $\begin{array}{c}\text { 5- Flowered } \\
\text { inflorescence }\end{array}$ & $\begin{array}{c}\text { 6- Flowered } \\
\text { inflorescence }\end{array}$ \\
\hline TN74116 & $44.84^{\mathrm{a}}$ & $45.79^{\mathrm{a}}$ & $7.99^{\mathrm{b}}$ & $1.38^{\mathrm{c}}$ & $0.0^{\mathrm{c}}$ \\
$\mathrm{TN} 74238$ & $36.95^{\mathrm{a}}$ & $45^{\mathrm{a}}$ & $17.06^{\mathrm{b}}$ & $0.99^{\mathrm{c}}$ & $0.0^{\mathrm{c}}$ \\
$\mathrm{TN} 74197$ & $58.42^{\mathrm{a}}$ & $38.34^{\mathrm{b}}$ & $3.24 \mathrm{f}^{\mathrm{c}}$ & $0.0^{\mathrm{c}}$ & $0.0^{\mathrm{c}}$ \\
$\mathrm{TN} 74100$ & $42.19^{\mathrm{b}}$ & $52.82^{\mathrm{a}}$ & $4.99^{\mathrm{c}}$ & $0.0^{\mathrm{c}}$ & $0.0^{\mathrm{c}}$ \\
$\mathrm{TN} 74128$ & $61.67^{\mathrm{a}}$ & $34.72^{\mathrm{b}}$ & $3.05^{\mathrm{c}}$ & $0.56^{\mathrm{c}}$ & $0.0^{\mathrm{c}}$ \\
$\mathrm{TN} 74237$ & $55.03^{\mathrm{a}}$ & $38.91^{\mathrm{b}}$ & $5.69^{\mathrm{c}}$ & $0.37^{\mathrm{c}}$ & $0.0^{\mathrm{c}}$ \\
$\mathrm{TN74156}$ & $33.12^{\mathrm{a}}$ & $33.16^{\mathrm{a}}$ & $28.13^{\mathrm{a}}$ & $5.59^{\mathrm{b}}$ & $0.0^{\mathrm{c}}$ \\
TN74243 & $32.26^{\mathrm{a}}$ & $39.3^{\mathrm{a}}$ & $22.98^{\mathrm{bc}}$ & $5.46^{\mathrm{c}}$ & $0.0^{\mathrm{c}}$ \\
TN74239 & $20.21^{\mathrm{b}}$ & $33.11^{\mathrm{a}}$ & $37.87^{\mathrm{a}}$ & $8.4^{\mathrm{c}}$ & $0.41^{\mathrm{d}}$ \\
TN74120 & $70.83^{\mathrm{a}}$ & $29.17^{\mathrm{b}}$ & $0.0^{\mathrm{c}}$ & $0.0^{\mathrm{c}}$ & $0.0^{\mathrm{c}}$ \\
TN74250 & $34.89^{\mathrm{b}}$ & $52.27^{\mathrm{a}}$ & $11.78^{\mathrm{c}}$ & $1.06^{\mathrm{c}}$ & $0.0^{\mathrm{c}}$ \\
TN74161 & $32.98^{\mathrm{b}}$ & $47.58^{\mathrm{a}}$ & $18.49^{\mathrm{c}}$ & $0.95^{\mathrm{d}}$ & $0.0^{\mathrm{d}}$ \\
TN74231 & $30.27^{\mathrm{ab}}$ & $38.24^{\mathrm{a}}$ & $22.44^{\mathrm{b}}$ & $8.77^{\mathrm{c}}$ & $0.28^{\mathrm{c}}$ \\
\hline
\end{tabular}

Values with the same letters within rows are not significantly different according to LSD test at $5 \%$ probability level. 
and TN74239, PSs in inflorescence accounts for the highest percentage of flowers $(22.96,22.62$, $22.28,24.76$, and $29.57 \%$, respectively), followed by LGs of single and inflorescence types. In all the genotypes, flowers with TSs (both single flowers and inflorescences) reached the lowest values (Table 3).

Regarding flowers capable (LGs+MEs) and incapable (SRTs) of setting fruit, LGs+MEs recorded higher values than SRTs in all the genotypes. Significant differences were found between these two types of flowers ( $\mathrm{P} \leq 0.05)$, except for genotype TN74128. However, total SRTs accounted for a considerable number of flowers in the plant. Genotype TN74120 recorded the highest percentage of LG+ME and the lowest percentage of total SRT $(75.62 \%$ and $20.47 \%$, respectively). Conversely, genotype TN74128 recorded the lowest percentage of $\mathrm{LG}+\mathrm{ME}$, and the highest percentage of SRT $(45.51 \%$ and $23.50 \%$, respectively) (Table 4 ).

\section{Initial fruit set based on style length}

There was a close relationship between initial fruit set and style length in eggplant genotypes under study. For both single flowers and inflorescences, LGs recorded high fruit set rates, with mean values of $71.40 \%$ and $45.66 \%$, respectively. Similarly, MEs also recorded high initial fruit set rates, except for genotype TN74120 in inflorescence. On the contrary, PSs (single flowers and inflorescences) presented low fruit set rates, while TSs resulted in $0 \%$ fruit set, with $100 \%$ abortion in all the genotypes (Table 5).

In all genotypes, the total percentage of initial fruit set of LG+ME was high, while a very low percentage of total SRTs (lower than $4 \%$ ) could set fruit. Genotype TN74120 and TN74161 recorded the highest and lowest percentage of initial fruit set of LG+ME, respectively (Table 4).

\section{Yield and style length}

Genotype TN74239 recorded the highest number of fruit and final yield (21 fruits per plant and $4023 \mathrm{~g}$, respectively), with no significant differences $(P>0.05)$ with some other genotypes, while genotype TN74116 recorded the lowest values (13.87 fruits per plant and $2410 \mathrm{~g}$, respectively).

Although there is a positive and significant correlation between flowers capable of setting fruit (LG+ME) and initial fruit set rates, there is a negative and significant correlation between SRT and initial fruit set rates (Table 6). However, no significant correlation was found between final fruit set or final yield and style length (long, medium and short).

\section{Pedicel size and style length}

There was a relationship between pedicel size (length and thickness) and style length. Table 7 shows pedicel length and thickness for the three types of flowers in all the genotypes studied. The highest values were observed in LGs and MEs in all the genotypes, while SRTs recorded lower values than those observed in the other two types of flowers. Average values for pedicel length were 2.52, 2.35 and $1.57 \mathrm{~mm}$, and for pedicel thickness were 2.97, 2.88 and $1.58 \mathrm{~mm}$ in LGs, MEs and SRTs, respectively.

Table 3. Percentage of single flowers and inflorescences in 13 eggplant genotypes. LG (Long-styled flower), ME (Medium-styled flower), PS (Pseudo short-styled flower), and TS (True shortstyled flower).

\begin{tabular}{|c|c|c|c|c|c|c|c|c|}
\hline \multirow{2}{*}{ Genotype } & \multicolumn{2}{|c|}{ LG (\%) } & \multicolumn{2}{|r|}{ ME (\%) } & \multicolumn{2}{|c|}{ PS (\%) } & \multicolumn{2}{|c|}{ TS (\%) } \\
\hline & Single & Inflorescence & Single & Inflorescence & Single & Inflorescence & Single & Inflorescence \\
\hline TN74116 & $17.25^{\mathrm{b}}$ & $19.02^{b}$ & $10.68^{c}$ & $12.95^{c}$ & $2.04^{\mathrm{d}}$ & $22.96^{\mathrm{a}}$ & $1.31^{\mathrm{d}}$ & $2.74^{\mathrm{d}}$ \\
\hline TN74238 & $46.80^{\mathrm{a}}$ & $11.62^{\mathrm{bc}}$ & $3.97^{\mathrm{d}}$ & $1.25^{\mathrm{d}}$ & $8.66^{\mathrm{cd}}$ & $15.95^{\mathrm{b}}$ & $2.13^{\mathrm{d}}$ & $0.73^{\mathrm{d}}$ \\
\hline TN74197 & $28.53^{a}$ & $25.35^{\mathrm{a}}$ & $5.81^{\mathrm{cd}}$ & $5.01^{\mathrm{c}}$ & $3.90^{c-e}$ & $18.61^{\mathrm{b}}$ & $0.88^{\mathrm{e}}$ & $1.69^{\mathrm{de}}$ \\
\hline TN74100 & $25.67^{a}$ & $10.04^{\mathrm{d}}$ & $21.35^{b}$ & $8.73^{\mathrm{de}}$ & $5.98^{\mathrm{d}}$ & $15.23^{c}$ & $2.50^{\mathrm{e}}$ & $2.35^{\mathrm{e}}$ \\
\hline TN74128 & $20.4^{\mathrm{a}}$ & $8.54^{\mathrm{c}}$ & $11.08^{\mathrm{c}}$ & $3.56^{\mathrm{d}}$ & $15.33^{b}$ & $22.62^{\mathrm{a}}$ & $5.15^{\mathrm{d}}$ & $2.39^{\mathrm{d}}$ \\
\hline TN74237 & $14.13^{\mathrm{b}}$ & $8.67^{c}$ & $19.78^{\mathrm{a}}$ & $12.21^{\mathrm{b}}$ & $7.67^{c}$ & $22.28^{\mathrm{a}}$ & $3.18^{\mathrm{d}}$ & $2.81^{\mathrm{d}}$ \\
\hline TN74156 & $34.07^{a}$ & $22.01^{\mathrm{b}}$ & $4.50^{\mathrm{d}}$ & $4.09^{\mathrm{d}}$ & $2.78^{\mathrm{d}}$ & $14.78^{\mathrm{c}}$ & $1.35^{\mathrm{d}}$ & $0.97^{\mathrm{d}}$ \\
\hline TN74243 & $20.2^{a}$ & $14.13^{\mathrm{b}}$ & $9.94^{\mathrm{bc}}$ & $7.78^{\mathrm{cd}}$ & $3.72^{\mathrm{de}}$ & $24.76^{a}$ & $2.14^{\mathrm{e}}$ & $1.84^{\mathrm{e}}$ \\
\hline TN74239 & $15.96^{\mathrm{b}}$ & $12.4^{c}$ & $7.05^{\mathrm{d}}$ & $6.22^{\mathrm{d}}$ & $3.58^{\mathrm{e}}$ & $29.57^{a}$ & $1.13^{\mathrm{e}}$ & $1.71^{\mathrm{e}}$ \\
\hline TN74120 & $56.24^{\mathrm{a}}$ & $7.73^{b}$ & $10.17^{b}$ & $1.46^{\mathrm{b}}$ & $7.73^{b}$ & $8.36^{\mathrm{b}}$ & $3.43^{\mathrm{b}}$ & $0.93^{\mathrm{b}}$ \\
\hline TN74250 & $34.84^{\mathrm{a}}$ & $19.54^{\mathrm{b}}$ & $8.82^{\mathrm{d}}$ & $4.97^{\mathrm{de}}$ & $5.22^{\mathrm{de}}$ & $14.84^{\mathrm{c}}$ & $1.94^{\mathrm{e}}$ & $1.49^{\mathrm{e}}$ \\
\hline TN74161 & $34.56^{\mathrm{a}}$ & $16.80^{\mathrm{b}}$ & $3.76^{\mathrm{cd}}$ & $2.69^{d}$ & $7.51^{\mathrm{c}}$ & $17.70^{\mathrm{b}}$ & $2.01^{\mathrm{d}}$ & $2.94^{\mathrm{d}}$ \\
\hline TN74231 & $26.67^{a}$ & $23.96^{\mathrm{a}}$ & $4.24^{c}$ & $5.61^{\mathrm{c}}$ & $2.39^{c}$ & $18.44^{\mathrm{b}}$ & $1.11^{\mathrm{c}}$ & $2.59^{c}$ \\
\hline
\end{tabular}

Values with the same letters within rows are not significantly different according to LSD test at $5 \%$ probability level. 
Table 4. Percentage of total LG+ME and PS+TS (SRT), percentage of initial fruit set, final fruit set and final yield of 13 eggplant genotypes. LG (Long-styled flower), ME (Medium-styled flower), PS (Pseudo short-styled flower), and TS (True short-styled flower).

\begin{tabular}{|c|c|c|c|c|c|c|}
\hline \multirow{2}{*}{ Genotype } & \multicolumn{2}{|c|}{ Percentage of flowers } & \multicolumn{2}{|c|}{ Percentage of initial fruit set } & \multirow{2}{*}{$\begin{array}{l}\text { Final fruit } \\
\text { set } \\
\text { (No/plant) }\end{array}$} & \multirow{2}{*}{$\begin{array}{c}\text { Final } \\
\text { yield } \\
\text { (g/plant) }\end{array}$} \\
\hline & $\mathrm{LG}+\mathrm{ME}$ & PS+TS (SRT) & $\mathrm{LG}+\mathrm{ME}$ & PS+TS (SRT) & & \\
\hline TN74116 & $59.91^{\mathrm{d}-\mathrm{f}}$ & $29.07^{\mathrm{j}-1}$ & $66.32^{\mathrm{bc}}$ & $2.19^{g}$ & $13.87^{\mathrm{b}}$ & $2410^{b}$ \\
\hline TN74238 & $63.66^{\text {bee }}$ & $27.49^{\mathrm{kl}}$ & $54.79^{\mathrm{de}}$ & $0.24^{\mathrm{g}}$ & $20.41^{\mathrm{a}}$ & $3591^{\mathrm{ab}}$ \\
\hline TN74197 & $64.71^{\mathrm{b}-\mathrm{d}}$ & $25.09^{1 \mathrm{~m}}$ & $72.14^{\mathrm{ab}}$ & $0.74^{\mathrm{g}}$ & $18.5^{\mathrm{ab}}$ & $3213^{\mathrm{ab}}$ \\
\hline TN74100 & $65.80^{\mathrm{bc}}$ & $26.07^{1 \mathrm{~m}}$ & $51.82^{\text {ef }}$ & $2.03^{g}$ & $17.56^{\mathrm{ab}}$ & $2799^{b}$ \\
\hline TN74128 & $43.60^{\mathrm{h}}$ & $45.51^{\mathrm{h}}$ & $61.74^{\mathrm{cd}}$ & $3.12^{\mathrm{g}}$ & $19.8^{\mathrm{a}}$ & $3787^{\mathrm{ab}}$ \\
\hline TN74237 & $57.30^{\mathrm{fg}}$ & $33.46^{j}$ & $63.41^{\mathrm{b}-\mathrm{d}}$ & $3.77^{\mathrm{g}}$ & $19.9^{\mathrm{a}}$ & $3701^{\mathrm{ab}}$ \\
\hline TN74156 & $60.37^{\mathrm{ch}}$ & $23.56^{\operatorname{lm}}$ & $69.87^{\mathrm{a}-\mathrm{c}}$ & $0.46^{\mathrm{g}}$ & $16.16^{\mathrm{ab}}$ & $3129^{\mathrm{ab}}$ \\
\hline TN74243 & $52.08^{\mathrm{g}}$ & $32.47^{\mathrm{jk}}$ & $67.49^{a-c}$ & $1.70^{\mathrm{g}}$ & $17.7^{\mathrm{ab}}$ & $2658^{\mathrm{ab}}$ \\
\hline TN74239 & $41.64^{\text {hi }}$ & $36.01^{\mathrm{ij}}$ & $70.38^{\mathrm{a-c}}$ & $0.67^{\mathrm{g}}$ & $21^{\mathrm{a}}$ & $4023^{a}$ \\
\hline TN74120 & $75.62^{a}$ & $20.47^{\mathrm{m}}$ & $77.25^{\mathrm{a}}$ & $0.0^{g}$ & $19.25^{\mathrm{ab}}$ & $3219^{a b}$ \\
\hline TN74250 & $68.19^{b}$ & $23.50^{\mathrm{lm}}$ & $45.77^{\text {ef }}$ & $1.45^{\mathrm{g}}$ & $18^{\mathrm{ab}}$ & $2787^{\mathrm{ab}}$ \\
\hline TN74161 & $57.82^{e-g}$ & $30.17^{j-1}$ & $42.59^{f}$ & $0.96^{g}$ & $20.8^{\mathrm{a}}$ & $3510^{\mathrm{ab}}$ \\
\hline TN74231 & $60.51^{\mathrm{ch}}$ & $24.54^{\mathrm{lm}}$ & $50.81^{\text {ef }}$ & $1.24^{\mathrm{g}}$ & $17.16^{\mathrm{ab}}$ & $3331^{\mathrm{ab}}$ \\
\hline
\end{tabular}

Values with the same letter for percentage of flowers, percentage of initial fruit set, final fruit set and yield per plant are not significantly different at $\mathrm{p}=0.05$

Table 5. Percentage of initial fruit set of all kinds of flowers in 13 eggplant genotypes. LG (Longstyled flower), ME (Medium-styled flower), PS (Pseudo short-styled flower), and TS (True short-styled flower).

\begin{tabular}{|c|c|c|c|c|c|c|c|c|}
\hline \multirow[t]{2}{*}{ Genotype } & \multicolumn{2}{|c|}{$\begin{array}{c}\text { Initial fruit set } \\
\text { LG (\%) } \\
\end{array}$} & \multicolumn{2}{|c|}{$\begin{array}{c}\text { Initial fruit set } \\
\text { ME (\%) } \\
\end{array}$} & \multicolumn{2}{|c|}{$\begin{array}{c}\text { Initial fruit set } \\
\text { PS (\%) } \\
\end{array}$} & \multicolumn{2}{|c|}{$\begin{array}{c}\text { Initial fruit set } \\
\text { TS (\%) }\end{array}$} \\
\hline & Single & Inflorescence & Single & Inflorescence & Single & Inflorescence & $\overline{\text { Single }}$ & Inflorescence \\
\hline TN74116 & $76.36^{\mathrm{ab}}$ & $55.41^{\mathrm{b}}$ & $82.86^{\mathrm{a}}$ & $55.39^{\mathrm{b}}$ & $13.88^{c}$ & $17.61^{\mathrm{c}}$ & $0.0^{c}$ & $0.0^{c}$ \\
\hline TN74238 & $59.38^{a}$ & $45.96^{a}$ & $48.83^{a}$ & $26.01^{b}$ & $3.33^{c}$ & $0.0^{c}$ & $0.0^{c}$ & $0.0^{c}$ \\
\hline TN74197 & $84.54^{\mathrm{a}}$ & $57.74^{\mathrm{b}}$ & $98.97^{a}$ & $57.41^{\mathrm{b}}$ & $0.0^{c}$ & $9.52^{c}$ & $0.0^{c}$ & $0.0^{c}$ \\
\hline TN74100 & $55.29^{a}$ & $41.50^{\mathrm{ab}}$ & $53.21^{a}$ & $45.52^{\mathrm{a}}$ & $6.94^{c}$ & $20.74^{\mathrm{bc}}$ & $0.0^{c}$ & $0.0^{c}$ \\
\hline TN74128 & $74.25^{\mathrm{a}}$ & $39.77^{b}$ & $63.77^{a}$ & $26.52^{\mathrm{bc}}$ & $19.09^{c}$ & $18.16^{c}$ & $0.0^{\mathrm{d}}$ & $0.0^{\mathrm{d}}$ \\
\hline TN74237 & $72.17^{a}$ & $41.69^{\mathrm{bc}}$ & $71.70^{\mathrm{a}}$ & $52.14^{\mathrm{ab}}$ & $20.17^{\mathrm{cd}}$ & $18.65^{\mathrm{d}}$ & $0.0^{\mathrm{d}}$ & $0.0^{\mathrm{d}}$ \\
\hline TN74156 & $78.56^{\mathrm{a}}$ & $59.53^{\mathrm{ab}}$ & $66.07^{\mathrm{ab}}$ & $50.15^{\mathrm{b}}$ & $0.0^{c}$ & $8.33^{c}$ & $0.0^{c}$ & $0.0^{c}$ \\
\hline TN74243 & $82.26^{\mathrm{a}}$ & $54.72^{\mathrm{b}}$ & $69.84^{\mathrm{ab}}$ & $53.53^{\mathrm{b}}$ & $17.77^{c}$ & $10.31^{c}$ & $0.0^{c}$ & $0.0^{c}$ \\
\hline TN74239 & $79.29^{a}$ & $65.57^{\mathrm{ab}}$ & $74.30^{\mathrm{a}}$ & $50.29^{b}$ & $13.33^{c}$ & $3.33^{c}$ & $0.0^{c}$ & $0.0^{c}$ \\
\hline TN74120 & $83.83^{a}$ & $37.50^{\mathrm{b}}$ & $85.45^{\mathrm{a}}$ & $0.0^{c}$ & $0.0^{c}$ & $0.0^{c}$ & $0.0^{c}$ & $0.0^{c}$ \\
\hline TN74250 & $60.26^{a}$ & $33.68^{b}$ & $33.63^{\mathrm{b}}$ & $18.38^{\mathrm{bc}}$ & $18.75^{\mathrm{bc}}$ & $0.0^{c}$ & $0.0^{c}$ & $0.0^{c}$ \\
\hline TN74161 & $51.88^{\mathrm{a}}$ & $27.14^{\text {bc }}$ & $30.35^{a-c}$ & $30.95^{\mathrm{ab}}$ & $8.33^{\mathrm{cd}}$ & $3.57^{\mathrm{d}}$ & $0.0^{\mathrm{d}}$ & $0.0^{\mathrm{d}}$ \\
\hline TN74231 & $70.21^{\mathrm{a}}$ & $33.41^{\mathrm{b}}$ & $66.32^{\mathrm{a}}$ & $46.46^{\mathrm{b}}$ & $13.09^{c}$ & $6.66^{c}$ & $0.0^{c}$ & $0.0^{c}$ \\
\hline Mean & 71.40 & 45.66 & 65.02 & 39.44 & 10.36 & 8.99 & 0.0 & 0.0 \\
\hline
\end{tabular}

Values with the same letters within rows are not significantly different according to LSD test at $5 \%$ probability level.

\section{DISCUSSION}

The results of this study showed that all the eggplant genotypes produced both single flowers and inflorescences. Pradeepa (2002) reported that most of the studied accessions $(80.3 \%)$ had both single flowers and inflorescences in the same plant. However, some of them $(16.0 \%)$ produced only inflorescences and a few (3.6\%) produced only single flowers. Moreover, Das et al. ( 2017) studied 27 cultivars of eggplant, reporting that 22 cultivars had both single flowers and inflorescences and others had only single flowers.

LGs, MEs and SRTs in the eggplant genotypes 
Table 6. Correlation between the percentage of flowering based on style length and initial fruit set, final fruit set and final yield. LG (Long-styled flower), ME (Medium-styled flower), PS (Pseudo short-styled flower), and TS (True short-styled flower).

\begin{tabular}{lccccc}
\hline & 1 & 2 & 3 & 4 & 5 \\
\hline 1. LG+ME (\%) & 1 & $-0.88^{* *}$ & $0.60^{*}$ & $-0.26 \mathrm{~ns}$ & $-0.47 \mathrm{~ns}$ \\
2. PS+TS (SRT) (\%) & & 1 & $-0.56^{*}$ & $0.36 \mathrm{~ns}$ & $0.48 \mathrm{~ns}$ \\
3. Initial fruit set (\%) & & & 1 & $0.30 \mathrm{~ns}$ & $-0.28 \mathrm{~ns}$ \\
4. Final fruit set (no/plant) & & & & 1 & $0.82^{* *}$ \\
5. Final yield (g/plant) & & & & & 1 \\
\hline
\end{tabular}

**: significant at $1 \%, *$ : significant at $5 \%$, ns: no significant

Table 7. Pedicel length and thickness of LG, ME and SRT in 13 eggplant genotypes, LG (Long-styled flower), ME (Medium-styled flower), and SRT (short-styled flower).

\begin{tabular}{llllllll}
\hline \multirow{2}{*}{ Genotype } & \multicolumn{3}{c}{ Pedicel length $(\mathbf{m m})$} & & \multicolumn{3}{c}{ Pedicel thickness $(\mathrm{mm})$} \\
\cline { 2 - 4 } \cline { 7 - 8 } & LG & ME & SRT & & LG & ME & SRT \\
\hline TN74116 & $4.16^{\mathrm{a}}$ & $3.83^{\mathrm{b}}$ & $1.66^{\mathrm{c}}$ & & $3.4^{\mathrm{b}}$ & $3.93^{\mathrm{a}}$ & $1.46^{\mathrm{c}}$ \\
TN74238 & $2.6^{\mathrm{a}}$ & $2.20^{\mathrm{b}}$ & $1.1^{\mathrm{c}}$ & & $2.98^{\mathrm{b}}$ & $3.30^{\mathrm{a}}$ & $1.09^{\mathrm{c}}$ \\
TN74197 & $2.6^{\mathrm{a}}$ & $2.41^{\mathrm{b}}$ & $1.4^{\mathrm{c}}$ & & $3.89^{\mathrm{a}}$ & $3.23^{\mathrm{b}}$ & $1.6^{\mathrm{c}}$ \\
TN74100 & $1.83^{\mathrm{b}}$ & $2.5^{\mathrm{a}}$ & $1.76^{\mathrm{c}}$ & & $3.03^{\mathrm{b}}$ & $3.36^{\mathrm{a}}$ & $1.43^{\mathrm{c}}$ \\
TN74128 & $2^{\mathrm{b}}$ & $2.5^{\mathrm{a}}$ & $1.16^{\mathrm{c}}$ & & $2.76^{\mathrm{a}}$ & $2.36^{\mathrm{b}}$ & $1.86^{\mathrm{c}}$ \\
TN74237 & $2.33^{\mathrm{a}}$ & $2.03^{\mathrm{b}}$ & $1.4^{\mathrm{c}}$ & & $3.77^{\mathrm{a}}$ & $3.2^{\mathrm{b}}$ & $1.66^{\mathrm{c}}$ \\
TN74156 & $2.5^{\mathrm{b}}$ & $3.4^{\mathrm{a}}$ & $1.83^{\mathrm{c}}$ & & $2.76^{\mathrm{a}}$ & $2.42^{\mathrm{b}}$ & $1.32^{\mathrm{c}}$ \\
TN74243 & $2.83^{\mathrm{a}}$ & $2.23^{\mathrm{b}}$ & $1.83^{\mathrm{c}}$ & & $2.86^{\mathrm{a}}$ & $2.26^{\mathrm{b}}$ & $1.86^{\mathrm{c}}$ \\
TN74239 & $2.33^{\mathrm{a}}$ & $2.33^{\mathrm{a}}$ & $1.83^{\mathrm{b}}$ & & $2.8^{\mathrm{b}}$ & $3.13^{\mathrm{a}}$ & $2.03^{\mathrm{c}}$ \\
TN74120 & $2.83^{\mathrm{a}}$ & $2.31^{\mathrm{b}}$ & $1.8^{\mathrm{c}}$ & & $2.4^{\mathrm{a}}$ & $2.26^{\mathrm{b}}$ & $1.33^{\mathrm{c}}$ \\
TN74250 & $2.43^{\mathrm{b}}$ & $2.5^{\mathrm{a}}$ & $1.8^{\mathrm{c}}$ & & $2.23^{\mathrm{b}}$ & $3^{\mathrm{a}}$ & $1.55^{\mathrm{c}}$ \\
TN74161 & $2.03^{\mathrm{a}}$ & $1.66^{\mathrm{b}}$ & $1.5^{\mathrm{c}}$ & & $2.56^{\mathrm{a}}$ & $2.1^{\mathrm{b}}$ & $1.33^{\mathrm{c}}$ \\
TN74231 & $2.33^{\mathrm{a}}$ & $2.1^{\mathrm{b}}$ & $1.5^{\mathrm{c}}$ & & $3.27^{\mathrm{a}}$ & $2^{\mathrm{b}}$ & $1.79^{\mathrm{c}}$ \\
Mean & 2.52 & 2.35 & 1.57 & & 2.97 & 2.88 & 1.58 \\
\hline
\end{tabular}

Values with the same letter for pedicel length and thickness are not significantly different at $\mathrm{p}=0.05$

averaged about 45,15 and $30 \%$, respectively, varying between the 13 genotypes under study. The ranges of total LGs, MEs and SRTs (single flower + inflorescence) were $22.8-63.97 \%$, 5.22$34.49 \%$ and $20.45-45.49 \%$, respectively. The results are in agreement with Mohideen et al. (1977), who reported similar values in eggplant cultivars: $25-$ $49 \%$ for LGs, $6.1-31.7 \%$ for MEs and $19.3-58.7 \%$ for SRTs. Previous studies also revealed that among all types of flowers in eggplant, flowers with high style are the largest group (over 60\%), while MEs and SRTs account for $10-20 \%$ and $20-30 \%$ of flowers, respectively (Passam and Bolmatis, 1997; Kowalska, 2003; Kowalska, 2006). Moreover, Nagasawa et al. (2001) and Pohl et al. (2019) reported that LGs and MEs often occur in higher number than SRTs.

According to the results obtained in the present study, there was a close relationship between initial fruit set and style length in the eggplant genotypes under study. Initial fruit set of LGs and MEs was high in both single flowers and inflorescences but too low in PSs, while TSs were not fertile or capable of setting fruits. These results are in agreement with Chen (2001) and Kowalska (2003), who reported higher fruit set rates from LGs and MEs rather than SRTs. Pandit et al. (2010) and Pohl et al. (2019) also reported higher fruit set rates from LGs and MEs compared to those from non-reproductive flowers (SRTs).

Chadha and Saimbhi (1977) described that the failure of eggplant flowers to set fruit can significantly decrease fruit yield potential since a considerable number of eggplant flowers are short-styled ones. Contrary to these reports, our results showed that even though there is a positive correlation between the percentage of LGs+MEs and initial fruit set, there is also a 
negative correlation between the percentages of SRTs and initial fruit set. However, there was no significant correlation between these types of flowers and final yield. That fact that SRTs fall before they can set fruits (flower abscission) may account for this situation. It is important to note that even though LGs and MEs can set fruits, they cannot grow and turn to harvestable fruits since fruit abscission occurs before harvest time. Therefore, it seems that style length does not affect final yield or final fruit set.

Although no clear explanation has yet been put forth for the failure of SRTs to set fruits, their floral morphological features (style length relative to stamen level) have been claimed to be involved in their self-pollination failure (Rylski et al., 1984; Sekara and Bieniasz, 2008). Smith (1931) claimed that low pedicel thickness might lead to the drop of these flowers. Rylski et al. (1984) showed that, despite the fully fertile pollens of SRTs, no germination would occur on their stigma. Moreover, while the nodules in LG stigmas are reportedly well-developed and their permeable tissues contain large amounts of proteins, polysaccharides, and other nutrients to enrich them for pollen germination and fruit setting, SRT stigmas lack adequate amounts of nutrients. Thus, there is no capacity for pollen absorption and germination (Handique and Sarma, 1995; Rylski et al., 1984). Several studies have also been carried out to find out the genetic pattern of style length and fruit setting in eggplants. According to the observations of Peter and Singh (1973), the number of LGs and MEs is controlled by additive gene action. Weijun (1992) showed that two genes control style length. However, Khapre and Wanjari (1987) carried out some research on the fruiting pattern of eggplant and observed that this trait is controlled by four genes. Moreover, Ushijima et al. (2012) also found that four genes are related to heterostyly development in Linum grandiflorum plant.

\section{CONCLUSION}

In summary, although there was a close relationship between style length and initial fruit set, no significant correlation was found between style length and final fruit set in eggplant. This means the style length is probably not effective on final yield, and longer styled flowers are not indicative of more fruits per plant and yield. Therefore, in order to increase yield, strategies to prevent fruit drop, which is formed from LGs and MEs, may be more effective than increasing the proportion of LGs and MEs to SRTs.

\section{LITERATURE CITED}

Caruso, G., R. Pokluda, A. Sękara, A. Kalisz, A. Jezdinský, T. Kopta, et al. 2017. Agricultural practices, biology and quality of eggplant cultivated in Central Europe. A review. Horticultural Science 44(4):201-212

Chadha, M. L., and M. S. Saimbhi. 1977. Varietal variation in flower types in brinjal (Solanum Melongena L.). Indian Journal of Horticulture 34(4):426-29.

Chen, N. C. 2001. Eggplant Seed Production. AVRDC International Cooperators' Guide. Asian Vegetable Research and Development Center, Shanhua, Taiwan.

Das, A., M. K. Pandit, S. Bairagi, S. Saha, and K. Muthaiah. 2017. A study on floral morphology of brinjal genotypes in gangetic-alluvial zone of west bengal, India. International Journal of Current Microbiology and Applied Sciences 6(10):3323-3331.

FAOSTAT. 2019. Agriculture Organization of the United Nations statistics division. Production Available in http://Faostat3. FAO. Org/Browse/Q/QC/S. Accessed December 22, 2020.

Handique, A. K., and A. Sarma. 1995. Alteration of heterostyly in Solanum melongena L. through gamma-radiation and hormonal treatment. Journal of Nuclear Agriculture and Biology 24(2):121-26.

Hazra, P., J. Manda, and T. P. Mukhopadhyay. 2003. Pollination behaviour and natural hybridization in Solanum melongena L., and utilization of the functional male sterile line in hybrid seed production. Capsicum and Eggplant Newsletter 22:143-46.

Jagatheeswari, D. 2014. Morphological studies on flowering plants (Solanaceae). International Letters of Natural Sciences 15:36-43.

Khapre P. R, K. B. Wanjari, and A. B. Deokar. 1987. Inheritance of fruiting pattern in eggplant. Journal of Maharashtra Agricultural Universities 12:3 401.

Kowalska, G. 2003. The influence of heterostyly, pollination method and hormonization on eggplant's (Solanum melongena L.) flowering and fruiting. Acta Agrobotanica 56(1-2):6176.

Kowalska, G. 2006. Eggplant (Solanum melongena L.) flowering and fruiting dynamics depending on pistil type as well as way of pollination and flower harmonization. Folia Horticulturae 18(1):17-29. 
Krishnamurtbi, S., and D. Subramanian. 1954. Some investigations on the types of flowers in brinjal (Solanum melongena), based on style length, and their fruit-set under natural conditions and in response to 2, 4-diohlorophenoxy acetic acid as a plant growth-regulator. Indian Journal of Horticulture 11(2):63-68.

Mohideen, M. K., C. R. Muthukrishnan, A. Rajagopal, and V. Asok Metha. 1977. Studies on the rate of flowering, flower types and fruit set in relation to yielding potential of certain eggplant (Solanum melongena L.) varieties with reference to weather conditions. South Indian Horticulture 25:56-61.

Nagasawa, M., A. Sugiyama, H. Mori, K. Shiratake, and S. Yamaki. 2001. Analysis of genes preferentially expressed in early stage of pollinated and parthenocarpic fruit in eggplant. Journal of Plant Physiology 158(2):235-40.

Pandit, M. K., H. Thapa, S. Akhtar, and P. Hazra. 2010. Evaluation of brinjal genotypes for growth and reproductive characters with seasonal variation. Journal of Crop and Weed 6(2):31-34.

Passam, H. C., and A. Bolmatis. 1997. The influence of style length on the fruit set, fruit size and seed content of bubergines cultivated under high ambient temperature. Tropical Science 37:221-27.

Peter, K. V., and R. D. Singh. 1973. Diallel analysis of economic traits in brinjal. Indian Journal of Agricultural Science 43(5):452-55.

Pohl, A., A. Grabowska, A. Kalisz, and A. Sękara. 2019. Biostimulant application enhances fruit setting in eggplant-An insight into the biology of flowering. Agronomy 9:482.

Pradeepa, G. L. 2002. Fruit-setting behaviour in relation to floral morphology of eggplant (Solanum melongena.). Tropical Agricultural Research and Extension 5: 13- 16.

Prasad, D. N., and R. Prakash. 1968. Floral biology of brinjal (Solanum melongena L). Indian Journal of Agricultural Sciences 38(6):1053.

Raigón, M. D., J. Prohens, J. E. Muñoz-Falcón, and F. Nuez. 2008. Comparison of eggplant landraces and commercial varieties for fruit content of phenolics, minerals, dry matter and protein. Journal of Food Composition and Analysis 21(5):370-76.
Rashid, M. A., and D. P. Singh. 2000. A manual on vegetable seed production in Bangladesh. AVRDC-USAID-Bangladesh Project, Horticulture Research Centre, Bangladesh Agricultural Research Institute, Gazipur, Bangladesh..

Rylski, I., J. Nothmann, and L. Arcan. 1984. Differential fertility in short-styled eggplant flowers. Scientia Horticulturae 22(1-2):39-46.

San José, R., M. Plazas, M. Sánchez-Mata, M. Cámara, and J. Prohens. 2016. Diversity in composition of scarlet (S. aethiopicum) and gboma (S. macrocarpon) eggplants and of interspecific hybrids between $S$. aethiopicum and common eggplant (S. melongena)." Journal of Food Composition and Analysis 45:130-40.

Sękara, A., and M. Bieniasz. 2008. Pollination, fertilization and fruit formation in eggplant (Solanum melongena L.). Acta Agrobotanica 61(1):107.

Singh, A. K., R. Singh, S. Kumar, and G. Kalloo. 2006. Genetic diversity within the genus solanum (solanaceae) as revealed by RAPD markers. Current Science 711-16.

Smith, O. 1931. Characteristics associated with abortion and intersexual flowers in the eggplant. Journal of Agricultural Research 43(1):83.

Srinivas, G., A. H. Jayappa, and A. I. Patel. 2016. Heterostyly: A threat to potential fruit yield in brinjal (Solanum melongena L.). Advancements in Life Sciences 5:1211-1215.

Ushijima, K., R. Nakano, M. Bando, Y. Shigezane, K. Ikeda, Y. Namba, S. Kume, T. Kitabata, H. Mori, and Y. Kubo. 2012. Isolation of the floral morph-related genes in heterostylous flax (Linum grandiflorum): the genetic polymorphism and the transcriptional and post-transcriptional regulations of the $S$ locus. Plant Journal 69:317-33.

Weijun, Z. 1992. Inheritance of isozymes and morphological characters in the brinjal eggplant. Acta Genetica Sinica 19:423-29. 